\title{
Génération $Y$ et marketing RH : une approche générationnelle de la communication de recrutement en France
}

\section{Namoin Yao}

\section{(2) OpenEdition}

\section{Journals}

Édition électronique

URL : http://journals.openedition.org/communicationorganisation/3539

DOI : 10.4000/communicationorganisation.3539

ISSN : 1775-3546

Éditeur

Presses universitaires de Bordeaux

Édition imprimée

Date de publication : 1 décembre 2011

Pagination : $59-70$

ISBN : 978-2-86781-745-8

ISSN : $1168-5549$

\section{Référence électronique}

Namoin Yao, «Génération $Y$ et marketing $\mathrm{RH}$ : une approche générationnelle de la communication de recrutement en France », Communication et organisation [En ligne], 40 | 2011, mis en ligne le 01 décembre 2014, consulté le 10 décembre 2020. URL : http://journals.openedition.org/

communicationorganisation/3539; DOI : https://doi.org/10.4000/communicationorganisation.3539 


\title{
Génération $Y$ et marketing RH : une approche générationnelle de la communication de recrutement en France
}

\author{
Nomoin Yao'
}

Les stratégies médiatisées de recrutement des organisations économiques ont connu un réel essor dans la période où il était question des difficultés rencontrées sur le marché du travail pour combler leur besoin de main-d'œuvre (2000-2007). La publication des études prévisionnelles concernant le départ à la retraite de la génération du Baby-boom a conduit certaines entreprises à fortement médiatiser leurs politiques et pratiques de ressources humaines (RH). Certains professionnels évoquaient la raréfaction des profils sur le marché du travail et la guerre des talents pour justifier cette forte médiatisation. Ces deux phénomènes concomitants seraient la résultante de l'effet Papy-boom à en croire leurs propos ${ }^{2}$. Ces opérations de recrutement (journées de recrutement, évènements, jeux d'entreprise, tournois sportifs, etc.) sont dans la majorité des cas destinées aux jeunes diplômés (25-30 ans) comme le montre l'extrait suivant : «La Société générale recevait les jeunes diplômés bac + 2/3, les 18 et 19 septembre 2005, au Stade de France, à l'occasion de ses quatrièmes "Rencontres Emploi". [...] PricewaterhouseCoopers (conseil en entreprise et expertise comptable) a procédé de même, du 4 novembre au 14 décembre 2005, en organisant la troisième édition du Connectedthinking Tour. En prévision du recrutement de 700 personnes dont 500 jeunes diplômés, un bus a parcouru les grandes villes de France et accueilli les étudiants d'une vingtaine d'écoles et universités ${ }^{3} »$.

\footnotetext{
1. Namoin Yao. Enseignante - Chercheur à l'Université de Lomé. Membre associé de l'EA 4426 Médiation Information Communication Art (MICA). Université Michel de Montaigne Bordeaux 3 ; ynamoin@yahoo.fr 2. Plus de cinq millions de personnes s'apprêtent à quitter leur entreprise ou leur administration dans la décennie à venir annonçait l'article "les entreprises en plein Papy-boom », L'Expansion, ler juin 2002, en ligne, page consultée le 20 septembre 2010, http://www.lexpansion.com/economie/les-entreprises-enplein-papy-boom_19128.html

3. Studyrama, en ligne, page consultée le 10 mars 2007, http://www.studyrama.com
} 
Cette segmentation générationnelle s'expliquerait-elle par les discours de la communication de recrutement et du marketing des ressources humaines centrés sur les meilleurs candidats?

Cet article, issu d'un précédent travail ${ }^{4}$, a une visée empirique et descriptive. La réflexion a été inscrite dans l'approche processuelle de la communication des organisations lors de cette recherche (Weick, 1969 ; Bonneville et Grosjean, 2007). À cet égard, nous nous sommes intéressée à l'ensemble des processus communicationnels ayant contribué à forger les notions de "difficultés d'embauche ", de "raréfaction de main-d'œuvre ", du Papy-boom et du " marketing des ressources humaines » en matière de recrutement. Les publications des auteurs comme Chantal Nicole-Drancourt, Laurence Roulleau-Berger (2006) et Laurence Fresne (2003) ont permis la prise en compte des dimensions sociologiques (âge, diplôme et situation géographique) compliquant la question de l'insertion professionnelle juvénile sur le marché du travail français. Nous nous sommes appuyée sur les critiques formulées à l'encontre des pratiques professionnelles de la fonction " communication " au sein des entreprises (Carayol, 2004 ; Le Moënne, 1999 ; Bernard, 1999 ; De Crescenzo et Floris, 2000 ; D'Almeida, 2001 ; Calais, 2003) et envers la communication managériale (Floris, 1996) pour assimiler la communication de recrutement à une communication institutionnelle managériale à cause de ces caractéristiques ${ }^{5}$ (Yao, $2011: 47$ ).

Ceci étant, ici, les différentes approches théoriques qui sous-tendent notre travail ne seront plus largement exposées ni discutées ${ }^{6}$. Ce texte expose certains résultats de cette recherche en analysant la prise en compte du public « jeune » au sein des stratégies communicationnelles du marketing $\mathrm{RH}$ en retenant le point de vue des professionnels du domaine. Les représentations qu'ont les responsables RH des candidats « jeunes " influencent-elles les stratégies de communication à leur destination ? Notre contribution s'appuie sur l'approche constructiviste de la communication (Mucchielli et Noy, 2005) et veut répondre à cette question en montrant la manière dont leurs représentations influencent leurs stratégies communicationnelles et les conduisent à l'organisation d'évènements spécifiques pour les jeunes diplômés.

Sur le plan empirique, une enquête qualitative a été menée auprès de vingt-trois responsables des ressources humaines faisant partie des entreprises classées comme les plus attractives pour les futurs managers et

4. Yao Namoin, La communication de recrutement: étude des pratiques évènementielles, Thèse de doctorat en Sciences de l'Information et de la Communication, sous la dir. de Valérie Carayol, Université Michel de Montaigne Bordeaux 3, 2011.

5. Il s'agit de son rattachement à la direction des entreprises concernées, de l'importance de l'opinion des futurs et jeunes diplômés (plusieurs enquêtes sont réalisées sur la perception qu'ont les jeunes diplômés des entreprises), de la recherche de la conformité à l'intérêt général et de l'usage des méthodes de marketing dans la communication institutionnelle de recrutement.

6. Nous renvoyons les lecteurs à la consultation du travail cité précédemment. 
les futurs ingénieurs ${ }^{7}$. Cette étude empirique (entretiens semi-directifs) avait pour objectif d'interroger ces professionnels sur la perception qu'ils ont de la communication de recrutement et des pratiques évènementielles des entreprises en France. Compte tenu de leur statut et de leur rôle, il nous a paru pertinent de les retenir pour l'enquête. L'analyse de contenu thématique des résultats a été faite manuellement afin de favoriser une meilleure compréhension du sens des entretiens menés. Les résultats ayant montré la prédominance de la référence à la jeunesse ou à la Génération $Y$ comme destinataires de ces stratégies de marketing des ressources humaines dans le discours des professionnels interrogés, nous nous baserons sur ceux-ci pour élucider nos propos. Quelques articles parus dans la presse professionnelle ou sur Internet nommant les jeunes diplômés comme étant la cible de ces stratégies de communication seront également cités.

\section{Les implications de la communication de recrutement et du marketing des ressources humaines}

Peu de publications en Sciences de l'information et de la communication (SIC) se sont intéressées à l'évolution récente de la communication de recrutement (Pablo, 2010). Pour ceux qui l'ont étudiée, elle est analysée comme une forme de publicité institutionnelle au cours de laquelle l'entreprise ou l'organisation se sert de son image pour communiquer et recruter (Masquellier, 1995 ; Lavigne, 2003). Elle est aussi envisagée, par Axel Gryspeerdt, comme une modalité des relations publiques intégrée au sein de la communication institutionnelle de l'entreprise (Gryspeerdt, 2000 : 273).

Thierry Libaert, quant à lui, écrit : "Sachant que les salaires d'embauche sont globalement semblables au niveau des grandes entreprises, l'image de l'entreprise apparaît comme un paramètre déterminant de la séduction qu'elle opère sur le jeune diplômé. En conséquence, moins généraliste et plus sélective, la communication de recrutement conserve toute sa pertinence » (Libaert, 2005 : 15). D’après Nicole Hébert « L'image est l'une des raisons essentielles qui préside à l'orientation des jeunes-loups. Recruter est sans doute l'une des premières raisons pour lesquelles les dirigeants des firmes françaises envisagent une campagne de publicité d'entreprise " (Hébert, 1987 : 128). Présentée comme un processus aisé dans les ouvrages et manuels professionnels, l'image d'une organisation reste une « notion plurivoque et floue » et sa construction très complexe (Le Moënne, 2008 : 131).

Outre les SIC, des auteurs anglo-saxons et des publications outre-Atlantique dans le domaine des Sciences de gestion ont aussi démontré l'influence

7. Il s'agit du Top 50 des entreprises classées comme les plus attractives par les futurs ingénieurs et les futurs managers publié par l'Institut Trendence en 2009, www.trendence.com. Les responsables de recrutement des entreprises suivantes ont répondu à nos questions entre 2009 et 2010 : ADIDAS, ALSTOM, AREVA, BMW, COLAS, CREDIT AGRICOLE, DECATHLON, DELOITTE, EDF, EIFFAGE, HSBC, KRAFTFOODS, MAZARS, MORGAN STANLEY, ORANGE, PHILIPS, PUBLICIS CONSULTANT RH, SNCF, SOCIETE GENERALE, SUEZ ENVIRONNEMENT, THALES, UNILEVER, VEOLIA. 
de l'image institutionnelle de l'organisation dans la décision du candidat de postuler ou non à l'annonce d'offre d'emploi (Belt et Paolillo, 1982 : 107 ; Gatewood et al., 1993 : 416 ; Backhaus, 2004 : 116). D'après Anna Blackman (2006 : 368), l'utilisation des visuels, la mention de la carrière et des opportunités de développement, la référence à un parcours professionnel spécial favorisent le développement de l'image institutionnelle dans une annonce d'offre d'emploi. Cette image, une fois développée, permet d'attirer des candidats potentiels. Pour Gérard Ryan, Marcel Gubern et Inma Rodriguez, l'importance de l'image institutionnelle dans la conception de la publicité de recrutement augmente en raison des préoccupations du public et de sa demande. Celles-ci sont en lien avec la responsabilité sociale et la sensibilisation de l'entreprise par rapport aux problèmes de son environnement. Il est donc important, d'après ces trois auteurs, de construire une image de marque employeur qui réponde à ces préoccupations (2000).

Ces auteurs anglo-saxons utilisent le terme " advertising recruitment " (publicité de recrutement) pour qualifier la communication de recrutement. Elle a émergé au lendemain de la fin de la Seconde Guerre mondiale dans le but de répondre au changement rapide de l'environnement économique (Belt et Paolillo, op. cit : 105). Dès lors, deux phénomènes majeurs simultanés font leur apparition et conditionnent désormais la gestion des ressources humaines. Il s'agit d'une part de l'augmentation du niveau d'exigence des industries afin de recruter les meilleurs candidats (et être plus compétitives sur le marché) et, d'autre part, de l'accroissement de la qualification des candidats pour répondre à cette exigence (Peretti, 2011). Dès cet instant, les entreprises vont chercher à embaucher les meilleurs candidats et l'idée selon laquelle ils représentent leur « ressource essentielle » se propage rapidement. « Le succès d'une entreprise repose en définitive sur le type d'employés qui y travaillent, ce qui incite les entreprises à attirer et conserver les bons employés »(Blackman, op. cit. : 367). Pour attirer les meilleurs candidats, les entreprises utiliseront les annonces presse et les campagnes d'affichage pour se faire connaître auprès de leur public cible. Pour exemple, une campagne à destination des jeunes diplômés lancée par AREVA en 2006 : «Développez votre talent avec nos métiers de l'énergie $[\ldots]$ » vise à renforcer l'image d'AREVA en tant qu'employeur de référence. Elle est composée de sept visuels représentant de jeunes ingénieurs dans leur environnement industriel. Le respect de la procédure et la publicité de recrutement n'assurent pas toujours la réussite d'une embauche. D'après Laurence Firoben et Catherine Hirsch, le recrutement est « une activité à hauts risques où les erreurs sont lourdes de conséquences et où les procédures, méthodes et instruments d'évaluation à disposition, bien que fort nombreux, laissent le recruteur dans une cruelle incertitude, incapables qu'ils sont bien évidemment de garantir le succès » (Firoben et Hirsch, 2003: 127). Les critères aidant pour le choix d'un candidat au détriment d'un autre sont 
parfois arbitraires et subjectifs. Le recrutement est difficilement envisageable comme une activité objective (Eymard-Duvernay et Marchal, 1997).

Avec le développement d'Internet, les entreprises ont recours aux nombreuses possibilités offertes par ce média pour communiquer sur leur besoin de main-d'œuvre. L'usage d'Internet en matière de communication de recrutement contribue à augmenter leur visibilité sur le marché du travail et dans l'espace public (Zanda, 2005 ; Brigano, 2006). Elles utilisent également le hors-média pour communiquer sur leurs politiques et pratiques RH . Dans cette logique, l'objectif de la communication de recrutement a muté. De la publicité de recrutement, elle est passée au marketing des ressources humaines défini par Philippe Liger comme une nouvelle approche de la relation salarié/ entreprise qui « consiste à considérer les collaborateurs, présents ou potentiels, comme des clients, au sens le plus noble. Il s'agit d'appliquer la logique et les techniques du marketing et de la communication pour

- attirer des candidats, les recruter et bien les intégrer ;

- fidéliser des collaborateurs impliqués » (Liger, 2007: 9).

À sa suite, Serge Panczuk et Sébastien Point vont la définir comme « un nouvel état d'esprit fondé sur des techniques marketing adaptées aux ressources humaines pour que l'entreprise et sa DRH puissent se vendre, se fidéliser et se renouveler» (Panczuk et Point, 2008 : 4). Dans cette perspective, les agences spécialisées en communication et marketing RH conseillent aux entreprises de se doter d'une « image de marque employeur » afin d'attirer les meilleurs candidats. Celle-ci est considérée comme «le processus de construction d'une identité unique et remarquable de l'employeur [...] un concept de l'entreprise qui le distingue de ses concurrents ». Il s'agit d'une «stratégie ciblée sur le long terme pour diriger l'intention et les perceptions des salariés, des candidats potentiels, et les différentes parties prenantes comme une entreprise spéciale » (Backhaus et Tikoo, $2004: 502) .{ }^{8}$

Il devient alors évident que meilleurs candidats sous-entend «jeunes diplômés » ou « futurs diplômés » et ce pour deux raisons principales. Premièrement ces slogans publicitaires visent un public externe à l'entreprise et, deuxièmement, on observe rarement la médiatisation des stratégies de recrutement externes des personnes relevant d'une autre catégorie d'âge.

8. Ces définitions révèlent l'hégémonie du marketing « pour, par et dans l'entreprise " comme l'a formulé Françoise Bernard (op. cit : 55). Elles nous font penser aussi à la " gestion symbolique » et au « marketing des idées » dont fait part Bernard Floris dans son article (2001). D'après l'auteur, le marketing est devenu une démarche universelle de gestion des relations humaines et sociales au-delà du marché. II ne s'agit plus uniquement de la marchandisation des biens et des services car le marketing consiste aussi à la fabrication des idées et à leur vente de manière symbolique. En se référant à la proposition et à la critique de B. Floris, nous pouvons avancer à la suite de nos lectures et de nos observations que plusieurs idées peuvent alors se vendre en matière de marketing des RH : les meilleurs talents donnent un avantage compétitif et concurrentiel à l'entreprise ; le développement d'une image attractive permet de les séduire, et de les captiver ; l'occupation du territoire de la communication et la veille informationnelle même en période de non embauche permet de maintenir sa visibilité. 
Notons par ailleurs que les meilleurs candidats peuvent être également des salariés plus expérimentés travaillant dans d'autres entreprises. Ces derniers seront dénichés par les chasseurs de tête ou par les réseaux (informels, numériques, associations d'anciens, etc.). Cependant, depuis quelque temps, ce positionnement marketing et ces campagnes de recrutement médiatisées s'adressent à une génération particulière.

\section{Génération Y : entre mythe et réalité}

Le vocabulaire des professionnels de la communication de recrutement a vu émerger les termes Génération $Y$ ou Digital natives pour faire référence aux individus nés de la fin des années 1970 jusqu'au milieu des années 1990. Leur tranche d'âge varie entre 15 et 30 ans. Cette terminologie apparue dans les stratégies de communication pour recruter n'est pas le produit de travaux sociologiques. Pour Yannick Fondeur, «Le concept de la génération "Y" a été introduit dans le jargon du marketing et du management en référence à celui de la génération "X", qui désigne la génération immédiatement postérieure à celle du Baby-boom, souvent décrite comme manquant d'identité propre d'où le qualificatif "X" $"{ }^{9}$. Selon les consultants en communication des ressources humaines, la particularité de cette Génération $Y$ c'est sa naissance à l'ère d'Internet et des TIC. «La génération des "digital natives", cette population âgée de 14 ans à 30 ans, qui a grandi avec les nouveaux médias, est habituée aux innovations permanentes. Exploiter les nouvelles technologies de manière créative et surprenante, c'est pour l'entreprise, le moyen d'adresser aux jeunes le message qu'elle est capable d'être en phase avec son époque $»^{10}$. Les jeunes de cette génération sont devenus la cible des stratégies de communication de recrutement d'après le triple objectif (attirer, retenir et fidéliser les meilleurs talents) de la gestion des ressources humaines (Panczuk et Point, $2008: 3$ ).

En référence aux publications de Bernard Floris (op. cit. 2001 : 5), et au regard des pratiques actuelles du " marketing des ressources humaines ", nous pensons que la nécessité pour les entreprises de connaître la demande des jeunes diplômés et la planification des meilleurs moyens de la satisfaire, tout en recherchant un avantage potentiel de cet échange, croît sans cesse.

D'après les prévisions annoncées au début des années 2000, ces futurs diplômés devaient être moins nombreux et arriver progressivement sur le marché du travail au début des années $2000^{11}$. Par conséquent, ils ont fait, et font, l'objet de toutes les attentions de la part des recruteurs. Les professionnels de la communication de recrutement promeuvent l'idée selon laquelle « ceux

9. Entretien avec Yannick Fondeur (économiste et chercheur au Centre d'Éłude pour l'Emploi). Paru dans le magazine Entreprises et Carrières. "Jeunes et entreprises : les nouvelles connexions », n 919, du 9 au 15 septembre 2008, p. 35.

10. Dossier « Marketing social et marque employeur: où en sommes-nous ? »Personnel, $n^{\circ} 490$, juin 2008 , p. 46.

11. "Les métiers en 2015 : Impact du départ des générations du Baby-boom », Enquête de la Direction de I'Animation de la Recherche des Etudes et des Statistiques (DARES), décembre 2005, n50/1, 8 p. 
qui la comprendront le mieux, grâce à leurs conseils, seront à même d'attirer et de valoriser ses «talents». D'où la mobilisation d'outils de recrutement censés correspondre à cette cible : utilisation de Facebook, salons virtuels sur Second Life, Speed recruiting... ${ }^{12}$. " La connaissance de la demande des candidats et la maîtrise des stratégies pour la satisfaire peuvent-elles revenir à les fabriquer ?

\section{Les représentations liées à la jeunesse et à son embauche}

D'après nos observations empiriques, la prédominance de la référence à la « jeunesse » dans les discours professionnels nous paraît s'expliquer aussi par l'imaginaire collectif véhiculé à son propos dans le domaine du travail. L'indication de cette catégorie de la population induit un certain imaginaire du type : la jeunesse est pleine d'avenir ; être jeune c'est avoir plus de force de travail à vendre que ses aînés ; c'est avoir une forte capacité d'adaptation aux conditions modernes de travail, etc. Pour les entreprises, la mention de l'embauche des jeunes diplômés dans leur discours et leurs pratiques communicationnels leur donne l'image d'une organisation innovante, compétitive, moderne et qui intègre les jeunes dans le monde du travail selon les responsables des ressources humaines interrogés lors de notre étude. Ceci peut avoir une répercussion non négligeable sur leurs stratégies de communication si l'on tient compte du contexte actuel caractérisé par la crise économique et le fort taux de chômage des jeunes Français. Dans ce cas, mentionner qu'on les recrute peut permettre à l'organisation économique de construire ou maintenir son positionnement et/ou sa notoriété sur le marché du travail et dans l'espace public.

Ces raisons expliquent en partie, d'après les professionnels rencontrés, la facilité qu'ils ont à médiatiser leurs politiques et pratiques de recrutement envers les jeunes diplômés au détriment d'une tranche d'âge plus avancée. Ces logiques ont énormément influencé l'émergence des stratégies de communication médiatisées et ont fait apparaître le marketing des ressources humaines avec des opérations de recrutement atypiques ${ }^{13}$.

\section{Le marketing RH : une communication générationnelle sur mesure?}

Les résultats de notre recherche sur la communication de recrutement ont particulièrement démontré le lien existant entre les représentations des

12. Entretien avec Yannick Fondeur, op. cit., p. 35.

13. II s'agit essentiellement de la technologie du Tag utilisé par la Société Générale en 2006 et du forum emploi organisé en 2007 sur Second Life (univers virtuel) par AREVA, UNILOG, ALSTOM, CAPGEMINI et L'OREAL. Peuvent aussi être cités la campagne de recrutement médiatisée de la Société Générale dénommée «1 CDI en 1 jour ", les jeux d'entreprise présentés comme de réels outils de recrutement et les tournois sportifs. Ces opérations sont atypiques à cause de leur irrégularité (temporalité) et de leur singularité. Elles ont marqué une rupture dans les procédures de recrutement classiques (publication d'offre d'emploi, sélection des candidats, entretien d'embauche, etc.) grâce à leur hyper médiatisation et à la nouveauté du concept. 
professionnels et les évènements organisés ${ }^{14}$. Ils ont aussi corroboré les propos des auteurs précités concernant l'influence de l'image institutionnelle et le « marketing des idées ".

Les pratiques évènementielles sont pensées comme une forme de communication générationnelle et sur mesure au vu de nos observations et de l'interprétation des résultats des entretiens semi-directifs. Nous parlons de communication générationnelle car ces opérations s'adressent prioritairement aux jeunes diplômés issus de la Génération Y. À ce propos, lors de notre recherche, aucune autre population (adultes ou seniors) n'a été mentionnée comme destinataire de ces pratiques ni dans la revue de la littérature ni lors de l'enquête empirique ${ }^{15}$.

D'après l'analyse de contenu thématique des résultats des entretiens semi-directifs, les pratiques évènementielles issues du marketing des ressources humaines constituent une « communication sur mesure ». En effet, l'idée selon laquelle à chaque type de population correspond un évènement particulier est très répandue dans le corpus des entretiens menés. Les responsables de recrutement ont clairement déclaré qu'ils n'organisent pas le même type d'évènement à destination des ouvriers et des cadres; à chaque profession et catégories socio professionnelles recherchées correspond un type d'évènement particulier. Les extraits suivants le confirment : « Nous adaptons le fond, la forme de notre message ainsi que sa qualité en fonction de profils que nous recherchons [...]. C'est une communication avec un message adapté aux cibles car on ne s'adresse pas de la même manière à des profils qualifiés (ingénieurs, cadres, etc.) qu'à des profils moins qualifiés (ouvriers, techniciens, etc.). [...] Nous recrutons plus de $80 \%$ d'ingénieurs et, ce faisant, nous ne sommes pas dans la situation de sociétés qui recrutent des commerciaux et qui elles ont besoin d'être dans le ludique à cause du profil recherché. Le profil des commerciaux c'est justement qu'eux-mêmes, dans leur poste vont jouer ça avec leurs clients. Les gens qui viennent chez nous sont en général des gens intéressés par nos projets et qui seront plus intéressés par une visite d'entreprise, ou une conférence avec un orateur qui vient leur parler de l'innovation chez nous que par un Business Game».

Cet article confirme l'hypothèse selon laquelle les représentations des responsables $\mathrm{RH}$ concernant les candidats « jeunes » les conduisent à mettre en place des évènements censés leur correspondre. Dans son développement, la publicité de recrutement a abouti au marketing des $\mathrm{RH}$ avec une forte médiatisation des pratiques et politiques de recrutement des entreprises. Ces

14. Outre celles mentionnées précédemment, d'autres représentations telles que : pour recruter les jeunes d'aujourd'hui il faut sortir des sentiers battus, innover en utilisant les technologies actuelles pour les attirer, montrer son dynamisme et sa capacité d'adaptabilité à son environnement subsistent dans les discours des professionnels. C'est en partant de ces idées qu'ils mettent en place des opérations susceptibles de répondre aux attentes de ces jeunes diplômés.

15. Même les RDV Egalité Compétences organisés par la SNCF depuis 2006 sont destinés aux « jeunes issus des zones urbaines sensibles ». 
dernières se caractérisent par un fort positionnement générationnel juvénile pour deux raisons. La première, c'est la terminologie " meilleurs candidats ", évoquant des "futurs et jeunes diplômés » formés récemment. La seconde, c'est la conception de campagnes de recrutement qui leur sont spécialement destinées. La faiblesse des publications en SIC sur ce sujet rend l'effort de théorisation ardu.

En guise de conclusion, notons que les discours et les pratiques des responsables RH ne facilitent pas pour autant l'insertion professionnelle de tous les jeunes de la Génération Y. Des travaux et des publications portent sur le phénomène de précarité auquel est confrontée une catégorie de la jeunesse française ${ }^{16}$. Au-delà de cette Génération précaire, quels discours les responsables $\mathrm{RH}$ tiennent-ils envers les futurs diplômés en cette période de crise économique?

\section{BIBLIOGRAPHIE}

ALMEIDA N. D', Les promesses de la communication, Paris, PUF, « Sciences, Modernités, Philosophies », 2001, 263 p.

BACKHAUS K., « An exploration of corporate recruitment descriptions on monster. com », Journal of Business Communication, April 2004, 41, n², p. 115-136.

BACKHAUS K., TIKOO S., "Conceptualizing and researching employer branding », Career Development International, 2004, 9, n5, p. 501-517.

BELT J., PAOLILLO J., "The influence of corporate image and specificity of candidate qualifications on response to recruitment advertisement ", Journal of Management, 1982, 8, n¹, p. 105-112.

BERNARD F., "Communication organisationnelle et usages des sciences humaines et Sociales : Fragments de réflexion », dans LE MOËNNE C. (dir.), Communications d'entreprises et d'organisations, Rennes, PUR, 1999, p. 49-78.

BLACKMAN A., "Graduating students' responses to recruitment advertisements ", Journal of Business Communication, 2006, 43, n², p. 367-388.

BONNEVILLE L., GROSJEAN S. (dir.), Repenser la communication dans les organisations, Paris, L'Harmattan, "Communication des Organisations », 2007, 294 p.

BOUMAZA M., PIERRE E., «Des mouvements de précaires à l'unification d'une cause ", Sociétés contemporaines, 2007/1, n65. p. 7-25.

BRIGANO S., Communication RH. Quelles réalités, Paris, Éditions Liaisons, «Entreprises \& Carrières ", 2006, 311p.

CALAIS P., L'entreprise au-delà de l'économie : les enjeux de la communication d'entreprise dans un monde globalisé, Paris, Descartes \& Cie, 2003, 171 p.

16. BOUMAZA M., PIERRE E., " Des mouvements de précaires à l'unification d'une cause ", Sociétés contemporaines, 2007/1 n 65. pp. 7- 25. "Génération Précaire "Santé et travail. Le journal des professionnels de la santé au travail, n²7, juillet- août 2007, 8 p. 
CARAYOL V., Communication organisationnelle : une perspective allagmatique, Paris, L'Harmattan, 2004, 219 p.

DE CRESCENZO J.-CL., FLORIS B., « La gestion participative comme objet de communications organisationnelles ", dans DELCAMBRE P. (dir.), Communications organisationnelles : objets, pratiques, dispositifs, Rennes, PUR, 2000, p. 159-174.

Entreprises et Carrières, "Jeunes et entreprises : les nouvelles connexions ", 2008, n 919.

FLORIS B., La communication managériale. La modernisation symbolique des entreprises, Grenoble, PUG, 1996, 265 p.

FLORIS B., "Communication et gestion symbolique dans le marketing », Les enjeux, 2001, 12 p.

GATEWOOD R., GOWAN M., LAUTENSCHLAGER G., " Corporate image, recruitment image, and initial job choice decisions ", Academy of Management Journal, 1993, 36, n², p. 414-427.

"Génération Précaire »Santé et travail. Le journal des professionnels de la santé au travail, n'27, juillet- août 2007, 8 p.

HÉBERT N., L'entreprise et son image : publicité institutionnelle pourquoi et comment, Paris, Dunod, 1987, 205 p.

DARES. Enquête de la Direction de l'Animation de la Recherche des Etudes et des Statistiques, «Les métiers en 2015: Impact du départ des générations du Baby-boom », Décembre 2005, n 50.1. 8 p.

FIROBEN L., HIRSCH C., "Quand l'éthique interpelle nos pratiques de recrutement ", Revue Economique et sociale, 2003, n4, p. 127-133.

FRESNE FL., Les jeunes et l'emploi, Paris, La découverte, « Repères », 2003, 122 p.

GRYSPEERDT A., "Construire les relations publiques pour les comprendre et les analyser », Sciences de la Société, mai/octobre 2000, n50/51, p. 261-277.

LAVIGNE A., «Communication de recrutement à l'Université Laval», Communication E Organisation, 2003, n²4, p. 239-257.

«Zéro pointé sur l'emploi des seniors », Liaisons sociales, 2008, n96, p. 65-74.

EYMARD -DUVERNAY FR., MARCHAL E., Façons de recruter. Le jugement des compétences sur le marché du travail, Paris, Éditions Métailié, "Leçons de choses ", 1997, 243 p.

LE MOËNNE C. (dir.), Communications d'entreprises et d'organisations, Rennes, PUR, 1999, 235 p.

LE MOËNNE C., "L'organisation imaginaire? ", Communication E゚ Organisation, 2008, n³4, p. 131-152.

LIBAERT T., La communication d'entreprise, Paris, Économica, 2005, 112 p.

LIGER P., Le marketing des ressources humaines. Attirer, intégrer, et fidéliser les salariés, Paris, Dunod, 2007, 195 p.

MAQUELLIER D., La communication de recrutement et ses méthodes publicitaires, Thèse de doctorat en sciences et techniques communes : sciences de l'information, documentation, sous la dir. de Michel Martinache, Valenciennes, Université de Valenciennes, 1995, 406 p. 
MUCCHIELLI A., NOY C., Etudes des communications: Approches constructivistes, Paris, Armand Colin, 2005, 239 p.

NICOLE-DRANCOURT C., ROULLEAU-BERGER L., Insertion des jeunes en France, Paris, PUF, « Que sais-je ? ", 4e édition, 2006, 123 p.

PABLO M., " La Communication de recrutement dans le milieu hospitalier », Communication \& Organisation, 2010, n'37, p. 103-113.

PANCZUK S. POINT S., Enjeux et outils du marketing RH. Promouvoir et vendre les ressources humaines, Paris, Eyrolles, Éditions d'Organisation, "Ressources humaines ", 2008, 239 p.

PERETTI J.-M., Gestion des ressources humaines, Paris, Vuibert, $17^{\mathrm{e}}$ édition, 2011, 246 p.

RYAN G., GUBERN M., RODRIGUEZ I., " Recruitment advertising : the Marketing-human resource interface ", International Advances in economic research, May 2000, 6, n², p. 354-364.

WEICK E. K., The Social Psychology of Organizing, Reading, M.A, Addison-Wesley,

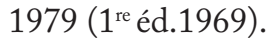

YAO N., La communication de recrutement : étude des pratiques évènementielles, Thèse de doctorat en Sciences de l'Information et de la Communication, sous la dir. de Valérie Carayol, Université Michel de Montaigne Bordeaux 3, 2011, 394 p.

ZANDA J.-L., Les employeurs qui recrutent par Internet, « Les essentiels », ANPE, 2005, $32 \mathrm{p}$.

Résumé : En France, les stratégies communicationnelles médiatisées des grands groupes multinationaux en matière de recrutement se sont beaucoup développées ces dernières années. Outre les entreprises, les organisations en général (universités, armées de terre, police, grandes écoles, hôpitaux, etc.) communiquent pour exprimer leur besoin de main-d'œuvre et pour accroître la visibilité de leurs politiques et pratiques de gestion des ressources humaines. Le vocable " marketing des ressources humaines " a vu le jour et sert à caractériser ces pratiques communicationnelles. L'observation et l'étude du phénomène révèlent deux enseignements : l'inscription de ces stratégies dans une approche « clientéliste » du recrutement et un positionnement marketing générationnel puisque ces stratégies de communication ont pour cible prioritaire les jeunes diplômés issus de la Génération $Y$.

Mots-clés : Communication, Génération Y, Marketing, Recrutement, Stratégies.

Abstract : In France, publicised communication strategies of big firms in recruitment have
greatly developed in recent years. Apart from firms, organizations in general (university, army,
police, high schools, hospital, etc.) communicate to express their need for labour and to increase
the visibility of their policies and human resource management practices. The term "marketing of
human resources"emerged and is used to characterize these communication practices. Observation
and study of the phenomenon show two things: the fitting of these communication strategies 
into the clientelist approach of recruitment, and, a generational marketing strategy since these communication practices have young graduates, who are Digital natives, as their target.

Keywords : Communication, Digital Natives, Marketing, Recruitment, Strategies. 